\title{
Engaged advocacy and learning to represent the self: Positioning people of color in our contemporary food movement
}

COMMENTARY ON RACE AND

ETHNICITY IN FOOD SYSTEMS WORK

\author{
Regina A. Bernard-Carreño* \\ Baruch College, City University of New York
}

Submitted June 15, 2015 / Published online September 21, 2015

Citation: Bernard-Carreño, R. A. (2015). Engaged advocacy and learning to represent the self: Positioning people of color in our contemporary food movement. Journal of Agriculture, Food Systems, and Community Development, 5(4), 189-193. http://dx.doi.org/10.5304/jafscd.2015.054.028

Copyright (C) 2015 by New Leaf Associates, Inc.

\begin{abstract}
Issues with access to food access are not solely that people of color are not included in the happenings of the food movement; it is also problematic just how our inclusion happens. Our issues within the movement are as diverse as we are, and there is no one particular narrative that can illustrate these sets of dilemmas easily. The solutions are even more difficult to generate and institute. Within these many complexities, however, both in addressing the problem and in finding positive results, there is also the problem of the lack of involvement within the community in a critical dialogue. Without a dialogue about these diverse sets of problems, working together to solve them seems a distant possibility.
\end{abstract}

\footnotetext{
* Regina A. Bernard-Carreño, PhD, Associate Professor, Black and Latino Studies, Baruch College, City University of New York; 55 Lexington Avenue, Suite 4274VC; New York, New York 10010 USA; profbernard.nyc@gmail.com
}

\section{Keywords}

food access, self-help, food sovereignty, people of color, culturally appropriate foods

\section{My Background in the Food System}

I was born in New York City of Guyanese heritage and am currently an associate professor in Black and Latino Studies at Baruch College, City University of New York. But anyone who knows me also knows that my personal narrative related to the food system usually begins with the phrase, "growing up in Hell's Kitchen in the 1970s." In my Hell's Kitchen neighborhood we had access to pizza, Chinese food, Afghan kebobs, and an enclosed farmers markets operating out of a parking garage. (It was not until the early 1990s that the neighborhood had its first sidewalk open-air farmers market that are now so commonly seen in the city.) We also had the choice of two major supermarkets: the A\&P supermarket (Atlantic \& Pacific Tea Co.), whose building is now a Citibank branch, and Red 
Apple Grocery, now Gristedes (another supermarket chain). Even with such a reasonable amount of access and despite people knowing how to cook and what to do with fresh vegetables, food struggles were not foreign to people in our neighborhood. Each month, west of our building a food pantry was operated in a church. The "cheese line," as it was called by the neighborhood children, was where extremely poor families acquired basic food staples. Although our family did not qualify for this food benefit, I had many schoolmates who stood on that line for hours for groceries like powdered milk, cheese, canned fruits in heavy syrup, potatoes, and other items. Receiving families would always share their food with families who did not qualify for the benefit. This provided a great opportunity to organize around the topic of food access. Families never complained about the quality of their food items, although sometimes the quantity was disputed between distributor and recipient. Accepting their free groceries without hesitation or further inquiry is an attitude toward food benefits that is also seen in recipients today.

However, in addition to the food offerings within short walking distances, I also had access to the tradition of a home-cooked meal, Guyanese style. Every day I watched my working mother come home from work and wash her hands before washing every leaf in a bunch of callaloo. She stripped potatoes of their skin with a knife, not a fancy peeler, and measured dry rice with her hands, not a measuring cup. She even soaked dry beans overnight after working a 40-hour-a-week job and attending school three nights a week. Every weekend while most children were outside playing, I and sometimes my older siblings accompanied my parents out on a food-shopping trip. Sure, there were cans of Campbell's soup in our shopping cart, but that was considered "emergency food." Every single day, my mother made a fresh and hot breakfast of eggs, toast, juice, and hot chocolate (on winter mornings).

\section{My Foray into Changing the Food System}

About a year ago I founded a farm-share program in Corona, Queens. It began very small, with just four members and an undergraduate student of mine alongside my husband and myself delivering the shares from Manhattan to Queens on a weekly basis. It was not long before I realized that families and other community members were not only unable to participate because of the price, but they had no real understanding of what a farm share meant as a larger community responsibility. In addition, farm shares and community supported agriculture (CSA) programs have been identified mostly in white, affluent communities and therefore are aligned with being one of "their" social programs of community rather than need. For lowincome communities, CSAs and similar program models are recent attempts to change the food access problem while signaling gentrification, a change that could mean their expulsion from the community. Shopping at the low-end chain supermarkets and the "fruit guy" at the train station's corner leads to a perception of access that seems to quell any desire for more by most residents. This version of access remains popular because many of the members in the community are not yet being given examples of how the entire neighborhood could be reignited, reawakened, revamped simply through the distribution of food by the person who actually grew it. There was and still is a gap in understanding why a pesticide-laden bunch of cilantro is different from the bunch that a $100 \%$ organic farmer offers.

In many communities that are lacking in better food choices, there is, no doubt, a lack of conversation as well. Among all their pressing and pending life issues, quality of food just is not ranking high enough on the list of important struggles. Residents of affluent communities already have access to successful schools and better opportunities for work. They have careers in place, not just jobs, and an overabundance of access to life's basic necessities. In low-income communities, these struggles stem from economic issues that rear their heads in every decision the family has to make, including food choices and whether or not critical thought is put into that decision each and every time food is consumed. This problem cannot be remedied by sending "organizers" into communities. There must be work on the part of the community itself to create its own organizers from among them. Only then can the problem be defined accurately and authentically. Only then can 
valid and unique solutions be found that address specific and diverse needs, rather just challenging global food access issues. Understanding the challenge requires an understanding of what set of information people have access to. The fight cannot be simply about making sure organic kale is in the Black neighborhood, but rather why it should be available in the Black neighborhood as well. What is the larger point behind having this access, rather than just increasing access? As we can see, simply bringing it to the community without discussion and inclusion leads to wilt, among the produce and the members of the neighborhood.

\section{Food Access Re-Evolution}

It is important to understand that food access for people of color has several layers, and these can be unpacked as we begin to regain our consciousness about our access and what we are eating on a regular basis. It is a common narrative in the larger public that people of colors' relationship to food is grounded in the overconsumption of fast food. However, many chefs of color are rewriting Black food history to reshape the dishes and recipes with more healthy ingredients. For some of us, if we were to consciously consider and study our Caribbean heritage, we would see that those diets historically were very green and largely about root vegetables. Critical but simple questions might arise, such as (1) if our diets were historically inclusive of spinach and sweet potatoes, then how come we are not eating them now? (2) Why can't we access them regularly? (3) Why are the big health-food stores monopolizing these groceries and making them largely unaffordable? (4) Why is there so much bureaucratic red tape in getting a farmers market to Black and Latino neighborhoods? (5) Why are we still very accepting of these circumstances?

The first step in the re-evolution has to be rethinking how we want to identify our relationship with food. There is a reason none of the fast-food restaurants in low-income Black and Latino neighborhoods are going out of business, but the libraries are losing funding. Perhaps through the redefinition of ourselves, we can change the dialogue in other groups about what they think they know about our history. Consider that the vegan diet, with its affluent subscribers, is the original diet of the Rastas. Yet veganism has become synonymous with Whiteness, affluence, and privilege, particularly in a big city like New York. We have become so dependent on television to teach families how to cook that we do not realize that those who are on TV mostly do not resemble us, and they use ingredients that we may have to work very hard to find. We have lost our will to ask the big and simple questions about the dishes that not only we are cooking, but that others are cooking and claiming as their own or as legitimate reinventions of the food wheel. It is really a stab in the heart to hear a beloved TV chef say, "You can find this in your local supermarket," and you know immediately that they are not saying this with you in mind. For if they did, they would know that a good portion of their viewers in low-income communities cannot access most of those basic ingredients, let alone tomato paste in a tube. What we need urgently are a new articulation of demand, a reintroduction to cooperation, and an updated model on collectively working for change. The need for valid inclusion in the food movement has to happen in steps, and has to have the very people being advocated for at the center of the table. How else can any of us know the true problems that need addressing?

As part of this first step we need to begin developing a new vocabulary that can critically define the existing problem, as opposed to using popular phrases that do not necessarily qualify a universal experience. For many of us living in a place like New York City, we are not struggling with food deserts. Actually, there is an overabundance of food in many of the neighborhoods that are identified as being "in trouble." The problem is that a lot of the food that is available should also be considered slow-kill poison; it is sold in the same aisle as pest killer at some of the neighborhood bodegas. If families make a proactive decision to skip past the burger chains, fried chicken restaurants, and, in some neighborhoods, ethnicspecific fast food, then the dependency immediately falls onto the neighborhood supermarket. The people who work at the supermarkets, both behind the register and stocking the shelves, are usually Black or Latino and are sometimes recently arrived 
immigrants. They reflect the shoppers in the community as well, so there is trust behind the purchasing experience. The owners however, are usually not from the neighborhood, do not speak the language of the shopper fluently, and are a different race altogether. They are marketing what they believe Black and Brown people use, eat, and enjoy. To some degree they must be right in their assessment, as items like canned food, frozen food, alcohol, salt, generic cold cuts (bits), soda, and fruit punch fly off the shelves. Many of the owners do not consider that the sincere "enjoyment" of these products comes from overconsumption and addiction to them, enabled by the very stores that sell them in plentitude. The fruits and vegetables at these supermarkets, when available, are waxy and shiny, and have colored lighting shining above them so they look fresh, farm-delivered, and ready to eat. It is not often you see a shopper at the local C-Town supermarket in Corona, Queens, using a smartphone to track the PLU code of a vegetable. There is not a single label in the supermarket that would be encountered in the supermarket of the affluent. No "non-GMO," "vegan-friendly," "allergen-free," "rBGH-free." But the time saved in not reviewing labels lets the shopper catch the bus and save a 20-minute walk home from the supermarket with heavy bags.

If we can begin to shift our vocabulary, we might be able then to engage in a better understanding of what we are actually accessing. The community most targeted for having a lack of options does not just need the option; it needs to rehabilitate the dialogue. The simple appearance of vegetables and fruit could signify gentrification. Everyone should be able to remain in their community but have access to quality food nearby as well. Healthy foods' appearance should not just be due to new, affluent residents.

The second step in the food access re-evolution is using critical dialogue about better access as a pedagogical tool. Simply placing a salad bar in a school cafeteria is not going to make long-term changes if the child cannot access a bunch of carrots outside of the cafeteria. The pedagogical tool that is designed must be useful for everyone in the community. Existing "community workshops" around the food issue(s) are usually introduced to low-income Black and Latino communities in a less-than-rewarding way. The same White, nonthreatening faces that are repeatedly cast as "food heroes" are the same faces that shows up to teach these communities about "eating properly" and how to consume fruits and vegetables. Adult members of families who are solely responsible for the food choices in their home do not want to be patronized, or reprimanded, and do not want to feel like they are being taught what is obviously best for their families, including their children. This is not to take away credit from programs that have the best of intentions in their outreach practices, but I have been witness to several surveyors and outreach coordinators who (1) do not understand the community they are reaching out to; (2) have no long-term investment in the community they are working in; and (3) take outreach to mean tacitly dehumanizing members of the community they are serving. People should be offered culturally appropriate tools to actively engage in the pursuit of their own change.

Aligned with the second step is the reshaping of our image as people of color and our involvement in the food system and food movement. We have become the visually illustrative example of the nation's "hungry." Having this label hanging over our experience with food does not invite humanizing opportunities for change. Neither does it beckon us to consider and research our cultural food history, and it certainly does not hold anyone else to a standard when invited to our communities to "advocate." Being identified as needy, be it due to unequal food access, inadequate or racist housing practices, or employment issues, detracts from the actual problem of inequality and economic disenfranchisement. Most of us in New York City and the outer boroughs, especially Queens, are not dying of starvation, although many of us fall weakness to atrocious food and are thus dying from our actual food consumption and choices. There is a desperate need to change our involvement in the food movement.

The third and final step of the re-evolution of food access is to promote leadership from within, even if it means alienating government officials, "do-gooders," and our neighbors. Brazilian philosopher Paulo Freire has always argued that the 
oppressed must wage their own war, and that their teachings to each other must also include teaching the oppressor as well. To teach the oppressor (whomever and whatever that representation) is to begin freeing the oppressed. To engage in change any other way, or to be dependent on anyone else for a glimpse of liberation, might be to engage in a struggle for a reward of which we do not really understand the value. Organizers who do not represent the communities that they advocate for and within cannot assume that everyone in those communities knows nothing about the change they wish to see. There are heroes and staunch community fighters living among those who are silent. If we are to own the re-evolution and lead in organizing, in defining change, and in articulating a positive act of inclusion, those heroes and fighters have to be actively sought out from our own ranks. 